\title{
APLICAÇÃO DE UM EPI INTELIGENTE EM ATIVIDADES QUE OFEREÇAM VIBRAÇÃO: UTILIZAÇÃO DE NOVAS TECNOLOGIAS
}

\author{
Beatriz Silva dos Passos Oliveira (UFCG) - beattrizoliveira@hotmail.com \\ Gilson da Silva Vital (UFCG) - gilsons628@gmail.com \\ Ingrid Rafaella Ferreira Duarte (UFCG) - ingridfduarte@ hotmail.com \\ Jefferson Teixeira de Souza (UFCG) - jefferson97ufcg@ gmail.com \\ José de Lima Henriques Júnior (UFCG) - jrhenrique01@gmail.com
}

\section{Resumo}

A ideia do uso de equipamentos de proteção individual tem sido, cada vez mais, descartada pelos trabalhadores em geral. São poucas as empresas que fornecem e exigem o uso correto de EPI's no ambiente de trabalho. Contudo, o empregado tem de ter entendimento das consequências que a falta dos equipamentos pode provocar, pois mesmo não evitando acidentes e/ou danos à saúde, podem proteger a incolumidade física do trabalhador durante a atividade laboral. Para tanto, pensou-se em um EPI inteligente com a função de medir a vibração localizada de mãos e braços- acoplado a uma luva antivibratória- que possa alertar o operário sobre o risco a que ele está submetido e a intensidade, visto que, o EPI mostrará ao trabalhador a que velocidade ele está utilizando a ferramenta e, caso ultrapasse o limite de tolerância o sensor emitirá sinais de alerta visual e auditivo. Devido a isso, o empregado se sentirá mais seguro e confiante em realizar sua tarefa.

Palavras-chaves: EPI; higiene e segurança do trabalho; vibração.

\section{Introdução}

O referente artigo tem por objetivo abordar a construção de um EPI inteligente que tem a função de medir a vibração localizada em mãos e braços em diferentes atividades, com a utilização do acelerômetro e giroscópio. Instrumentos esses que permitem uma nova interação com as máquinas e são responsáveis por medir e detectar vibrações e manter a orientação.

O monitoramento de atividades cada vez mais complexas dentro das empresas, exigem o uso de tecnologias de informação que facilitem cada vez mais o seu planejamento e que auxiliem na tomada de decisão, otimizando tempo, trabalho e mão de obra. Devido à concretização do 
mundo globalizado, há uma busca constante por melhorias tecnológicas para atender a necessidade de métodos de processamentos mais eficientes e rápidos. O acelerômetro é um exemplo dessas tecnologias, que possibilita a medição da intensidade repassando a informação para o programa.

A tecnologia pode ser instrumento de grande contribuição para facilitar, tornar ágil e eficiente a fiscalização e organização de tarefas, incluindo os aspectos de segurança do trabalho que a envolvem. As empresas de pequeno a grande porte devem seguir inúmeras regras de segurança do trabalho e o uso de tecnologias e inovações são de grande importância para auxiliar no desenvolvimento de soluções e produtos inovadores, a fim de garantir o cumprimento de leis e normas de segurança e manter o bem-estar dos funcionários.

\section{Fundamentação teórica}

\subsection{Higiene e Segurança do Trabalho}

Para melhor compreensão sobre o assunto a discutir, a higiene e segurança do trabalho pode ser apresentado e definido separadamente, de modo que Higiene do trabalho, de acordo com Saliba et al. (1997:11) é definida como:

“A ciência e a arte dedicada à antecipação, reconhecimento, avaliação e controle de fatores e ricos ambientais originados nos postos de trabalho e que podem causar enfermidade, prejuízos para a saúde ou bem-estar dos trabalhadores, tendo em vista o possível impacto nas comunidades e no meio ambiental em geral.”

E segurança do trabalho, pode ser entendida como Saliba (2011) a ciência dedicada na prevenção dos acidentes do trabalho, que são resultantes dos fatores de riscos operacionais no posto de trabalho.

A Higiene e Segurança do Trabalho relaciona-se a atividades que tem como finalidade: garantir melhores condições de trabalho e manter certo nível de saúde dos empregados. Onde a condição de trabalho pode ser entendida como uma variável presente no ambiente de trabalho que altera a produtividade do trabalhador, acometendo ou não a saúde do indivíduo. (BARBOSA FILHO, 2011).

Desse modo para atuar e garantir sua aplicação eficientemente é preciso entender os riscos, que cercam o indivíduo nos seus postos de trabalho, esses riscos podem ser classificados em cinco grupos de acordo com Barbosa Filho (2011):

- Riscos químicos, que representa os componentes presentes no campo de atuação da toxicologia (névoa, neblina, fumos, poeiras, gases e vapores); 
- Riscos biológicos, correspondes à capacidade de organismos vivos, chamados patogênicos (bactérias, fungos, helmintos, protozoários e vírus.);

- Riscos físicos, são caracterizados por aqueles que causam danos a partir de suas variáveis (ruído, vibração, temperaturas extremas, pressão anormal e radiação);

- Riscos de acidentes, que são consecutivos a partir da presença de materiais de oportunidades de danos (partes móveis de equipamentos, arestas cortantes, quinas vivas, entre outros);

- Riscos ergonômicos, decorrentes de diversas particularidades, que vão de uma inadequação antropométrica até as tarefas e informações que serão desenvolvidas pelo indivíduo.

Ao expor o indivíduo a algum desses agentes, acima do limite de tolerância estabelecidos na norma, cria-se uma insalubridade no trabalho, onde de acordo com a NR15 - art. 198.

\subsubsection{Riscos físico: vibração}

Tratando-se em específico os riscos físicos, as vibrações decorrentes do trabalho e seus danos para o indivíduo quando exposto acima do limite de tolerância.

Apesar do indivíduo estar frequentemente exposto a vibração nas indústrias, o estudo sobre vibrações não é tão significativo quanto os demais agentes, porém as consequências desse agente são altamente relevantes, por isso a importância de se realizar uma avaliação e controle mais rigorosos. (SALIBA, 2011).

A vibração pode ser considerada como uma oscilação de segmentos de um corpo em torno de determinado ponto fixo ou de referência (BARBOSA FILHO 2011).

E a quantidade de repetições dos ciclos durante um período de segundo, é determinado por frequência, com unidades de Hertz (Hz), os danos ao corpo do indivíduo dependem - dentre outras variáveis, como: amplitude do deslocamento ou a aceleração sofrida pelo corpo em movimento, sua direção e duração - dessa frequência, onde as mais danosas estão até $100 \mathrm{~Hz}$, denominadas de baixa frequência.

\subsubsection{Classificação da vibração}

Para determinar os danos à saúde decorrentes da exposição a vibração, é essencial definir a que tipo de vibração o trabalhador estará exposto. As vibrações podem ser classificadas, de acordo com Saliba (2011), como: 
- Vibração ocupacional do corpo inteiro, são as vibrações conduzidas para todo o corpo, em geral por meio de superfícies de suporte para o corpo;

- Vibração ocupacional de mão e braço ou localizada, são vibrações que atingem partes especificas, normalmente mão e braço;

- Vibração para conforto, são determinados por ocupações que geram desconforto intolerável, em certas situações e toleradas em outras;

- Vibração do meio ambiente, são vibrações que perturbam e desconforta a população ou grupo em uma área;

- Vibração de máquinas, são vibrações provenientes das maquinas utilizadas, que podem indicar problemas de manutenção.

Como normalmente vibrações, produzem também ruídos, ao adotar medidas preventivas para diminuí-los, acaba por reduzir aquele e vice-versa (BARBOSA FILHO, 2011).

\subsection{Exposição a vibração localizada}

Quando submetido a vibrações por longos períodos, seja no corpo inteiro ou em partes especializadas (mão, membros superiores ou inferiores), os danos à saúde do indivíduo, podem atingir o sistema circulatório, neurológico e ósteo-muscular, resultando na degradação dos mesmos, e com potencial danoso aos olhos, sistema digestivo e a coluna vertebral (BARBOSA FILHO, 2011).

\subsubsection{Vibração localizada ou mão e braço- danos}

De modo geral: os efeitos a este tipo de exposição podem ser vasculares, neurológicos, osteoarticular e muscular, sendo gradativo e passando por vários estágios de acordo com a frequência a ser exposto o indivíduo (SALIBA, 2011).

Para bloquear os possíveis danos, podem ser adotados como última tentativa a introdução de proteção individual, os EPI (Equipamento de Proteção Individual) - que serão tratados posteriormente- estes equipamentos devem ser disponibilizados só após a identificação e avaliação do risco (BARBOSA FILHO, 2011).

\subsection{Norma regulamentadora 15}

A legislação brasileira prevê, por intermédio da NR - 15 - Anexo 8, com redação dada pela Portaria n. 12 de 1983, que as atividades e operações que exponham os trabalhadores sem proteção adequada às vibrações localizadas ou de corpo inteiro serão caracterizadas como insalubres, apuradas por perícia realizada no local de trabalho. A perícia, visando à 
comprovação ou não da exposição, deve tomar por base os limites de exposição definidos pela Organização Internacional para a Normalização, em suas normas ISO 2.631 e ISSO 5.349 ou suas substitutas.

\subsubsection{Norma para avaliação da exposição ocupacional a vibrações em mãos e braços}

A Norma de Higiene Ocupacional, anexo 10 tem por objetivo estabelecer critérios e procedimentos para avaliação da exposição ocupacional a vibrações em mãos e braços que implique risco à saúde do trabalhador, entre os quais a ocorrência da síndrome da vibração em mãos e braços (SVMB).

Segundo Saliba (2011), a norma ISSO 5349/86 estabelece que as tomadas de medição das vibrações localizada ou de mão/braço, são ponderadas de terça de oitava nas faixas compreendidas entre $6,3 \mathrm{~Hz}$ a $1.250 \mathrm{~Hz}$, com fatores de ponderações distintos para cada frequência.

\subsubsection{Critério de avaliação da exposição ocupacional à vibração}

Para os fins desta norma, são utilizados os valores de referência descritos a seguir.

O nível de ação para a exposição ocupacional diária à vibração em mãos e braços adotado nesta norma corresponde a um valor de aceleração resultante de exposição normalizada (aren) de 2,5 $\mathrm{m} / \mathrm{s}^{2}$.

O limite de exposição ocupacional diária à vibração em mãos e braços adotado nesta norma corresponde a um valor de aceleração resultante de exposição normalizada (aren) de $5 \mathrm{~m} / \mathrm{s}^{2}$.

Para fins de comparação com o limite de exposição ou com o nível de ação, independentemente da duração da jornada de trabalho, deve- -se determinar a aceleração resultante de exposição normalizada (aren).

A aceleração resultante de exposição normalizada (aren) é determinada pela expressão:

$$
\text { aren }=\operatorname{are} \sqrt{\frac{T}{T_{0}}} \quad\left[\mathrm{~m} / \mathrm{s}^{2}\right]
$$

Sendo:

are $=$ aceleração resultante da exposição representativa da exposição ocupacional diária;

$\mathrm{T}=$ tempo de duração da jornada diária de trabalho, expresso em horas ou minutos;

T0 $=8$ horas ou 480 minutos 
Todas as acelerações consideradas neste critério são ponderadas em frequência, segundo a curva de ponderação Wh apresentada no Gráfico 1, conforme estabelecido no Anexo A da Norma ISO 5349-1: 2001 e especificada na ISO 8041 (2005).

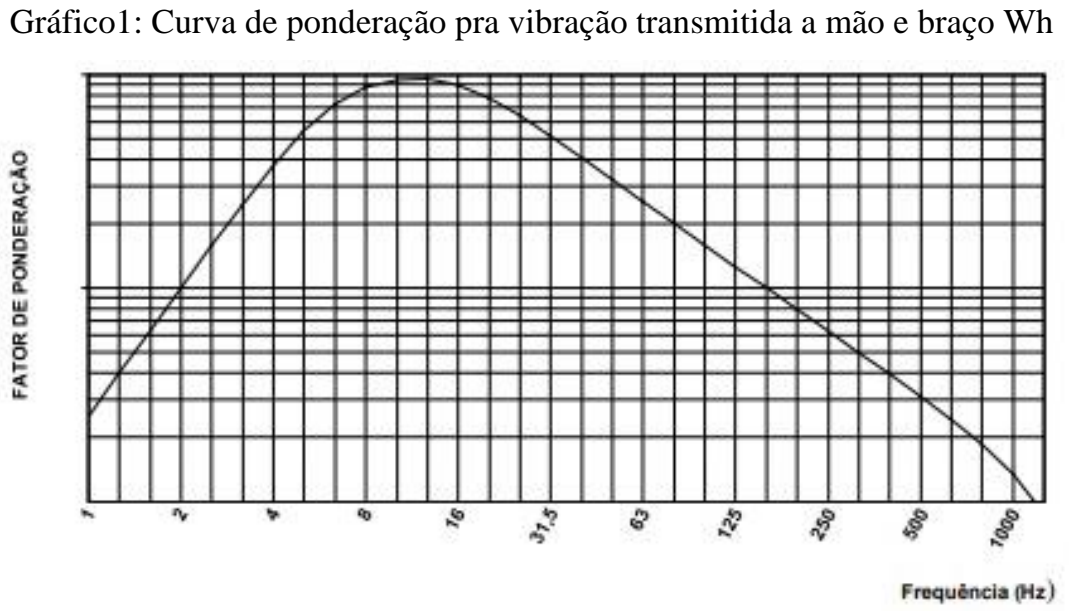

Fonte: Gerada a partir da ISSO 8041 (2005)

\subsubsection{Avaliação quantitativa da exposição- procedimentos gerais}

A avaliação da exposição ocupacional à vibração em mãos e braços deverá ser feita utilizandose de sistemas de medição que permitam a obtenção da aceleração resultante de exposição normalizada (aren), parâmetro representativo da exposição diária do trabalhador.

Os sistemas de medição devem ser compostos basicamente de medidores integradores e transdutores (acelerômetros) do tipo triaxial. Esses transdutores serão posicionados nos pontos de medição.

O conjunto de medições deve ser representativo das condições reais de exposição ocupacional do grupo de trabalhadores objeto do estudo. Desta forma, a avaliação deve cobrir todas as condições operacionais habituais e rotineiras que envolvem o trabalhador no exercício de suas funções.

\subsubsection{Equipamento de medição}

Para a realização das medidas, de acordo com Barbosa Filho (2011), são utilizados acelerômetros piezoelétricos (sensores e movimento), que exibe a aceleração que estão submetidos, fornecendo informações de grandeza mecânica em sinais elétricos. 
Segundo Saliba (2011), as tomadas de medidas devem ser realizadas de acordo com os procedimentos e a instrumentação especifica da norma ISO 5349, os equipamentos devem ter a capacidade de determinar a aceleração "rms" ponderada nos eixos em m/s2.

\subsection{Medidas para controle}

As medidas de controle devem ser adotadas tanto como medidas preventivas como corretivas, a fim de minimizar ou anular a repetição das situações que causem algum dano a saúde do trabalhador. Algumas medidas de controle adotadas para exposição a vibração são (Saliba, 2011):

- Usar ferramentas com características antivibratórias;

- Utilização de luvas antivibração;

- Execução de práticas adequadas de trabalho que permitem manter as mãos e o corpo do trabalhador aquecidos, bem como, minimizar o acoplamento mecânico entre o trabalhador e a ferramenta vibratória;

- Implantação de programas de supervisão médica.

\subsection{Equipamentos de proteção individual}

Os equipamentos de proteção individual, conhecidos pela sigla EPI, são definidos pela Norma Regulamentadora número 6 - NR 6, aprovada pela Portaria no 3.214/78, do Ministério do Trabalho, como:

"Todo dispositivo ou produto, de uso individual utilizado pelo trabalhador, destinado à proteção de riscos suscetíveis de ameaçar a segurança e a saúde no trabalho”.

O uso do EPI é de extrema importância, pois assegura a saúde do trabalhador durante a jornada de trabalho, protegendo-o individualmente contra possíveis riscos e consequências nocivas em caso de acidentes de trabalho.

A NR 6 determina que os EPIs sejam fornecidos de forma gratuita ao trabalhador para o desempenho de suas funções dentro da empresa. Além disso, é obrigatório que a empresa fiscalize, supervisione e oriente o funcionário quanto ao uso adequado dos equipamentos de proteção individual. Os EPI's devem ser utilizados durante toda a jornada de trabalho, cumprindo todas as exigências da empresa.

Vale ressaltar que o uso deste tipo de equipamento só deve ser utilizado quando não for possível minimizar os efeitos prejudiciais do ambiente de trabalho em que se desenvolve a atividade, como a utilização de equipamentos de proteção coletiva (EPCs), por exemplo. 
Para Saliba (2011) indicar corretamente o EPI é fundamental para garantir sua eficiência no controle do meio, exigindo critérios para a seleção do EPI, destacando-se:

a) A identificação e avaliação dos fatores de risco existentes nos ambientes de trabalho;

b) Com base na avaliação, deve-se selecionar o EPI mais adequado e que atenda as exigências.

Tendo outro aspecto importante que deve ser observado, a participação do trabalhador na escolha, visto que o usuário tem uma melhor avaliação do equipamento, havendo também como orientação para a escolha do EPI, o anexo I da NR6 que estabelece um quadro geral de orientação para cada EPI e suas respectivas aplicações de acordo com o fator de risco (SALIBA, 2011).

Assim como é necessário garantir o gerenciamento do uso do EPI, visto que é imprescindível como prova em ações jurídicas e processos administrativos, sendo como base para esse gerenciamento acatar as prescrições da NR-6.

\subsubsection{Tecnologia aplicada a EPI}

Com o uso da tecnologia aplicada ao EPI é possível além da proteção, prever ou informar situações de perigo e, consequentemente evitar e/ou minimizar os danos nos trabalhadores. Com alguns equipamentos eletrônicos é possível produzir um EPIN (Equipamento de Proteção Individual Inteligente), com baixo custo e com uma finalidade notável. Esses podendo ser acoplados diretamente no EPI, gerando uma função extra para o EPI, como:

- Placa Arduino: tem capacidade de receber informações (entradas: luz, sensor, dedo em um botão), e transformar em saídas (ativando um motor, ligando um LED), a partir de um conjunto de instruções envidas para o microcontrolador na placa, usando a linguagem de programação e o Software Arduino (IDE);

- Acelerômetro e giroscópio: sensores com 6 eixos, que informam exatamente para qual direção o objeto está se movendo, (devido aos 3 eixos do giroscópio) e medem a aceleração e intensidade aplicada ao objeto (medido a partir dos 3 eixos do acelerômetro), gerando uma informação que pode ser transmitida a partir de um programa;

- Display oled: compreendida como tela que transmite informação ao usuário, podendo ser utilizada em infinidade de aplicações, devido a sua variedade de tamanhos e facilidade de uso; 
- Led (diodo emissor de luz): componente eletrônico que emite luz, transformando energia elétrica em energia luminosa;

- Buzzer: dispositivo que produz um som (bipe), ou seja sinaliza áudio, que pode ser mecânico, eletromecânico ou piezoelétrico. Amplamente utilizado como dispositivos de alarme, temporizadores e confirmação de ações.

\section{Metodologia}

O presente artigo é uma pesquisa de caráter experimental e exploratório.

- Pesquisa experimental: "toma o próprio objeto em sua concretude como fonte e o coloca em condições técnicas de observação e manipulação experimental nas bancadas e pranchetas de um laboratório, onde são criadas condições adequadas para seu tratamento.” (ANTÔNIO SEVERINO, 2007);

- Pesquisa exploratória: "busca apenas levantar informações sobre um determinado objeto, delimitando assim um campo de trabalho, mapeando as condições de manifestação desse objeto.” (ANTÔNIO SEVERINO, 2007).

O projeto foi desenvolvido dentro da disciplina de Higiene e Segurança do Trabalho, no qual o objetivo a ser atingido foi a construção de um EPIN que mensure a vibração de máquinas, em determinada atividade, e emita um bip quando a vibração ultrapassar o limite de tolerância. Para tanto, objetivou-se aplicar os conceitos de metodologia de Projeto de Produto, e assim desenvolver um novo produto. Essa metodologia compreende as seguintes etapas:

Figura 01 - Etapas da metodologia

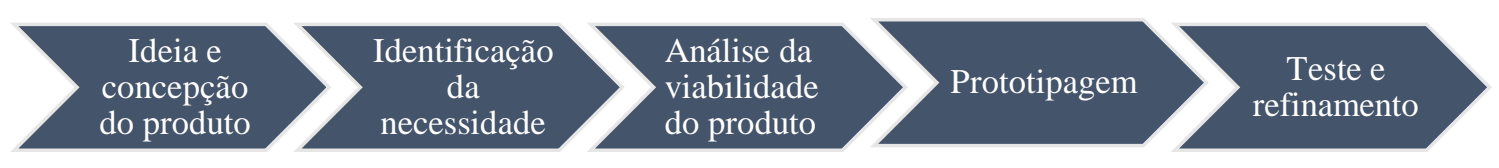

Fonte: Autoria própria (2018)

Nesse contexto, o Processo Desenvolvimento de Produtos (PDP) é considerado um conjunto de atividades que pretende, a partir das necessidades do mercado e das possibilidades e restrições tecnológicas, e considerando as estratégias competitivas e de produto da empresa, chegar às especificações de projeto de um produto e de seu processo de produção, trazendo resultados que atinjam o público-alvo e que os tornem viáveis para o processo de fabricação. (ROSENFELD et al., 2006). 
O processo de montagem do EPIN foi desenvolvido no laboratório de automação, da Universidade Federal de Campina Grande (UFCG) - campus Sumé, com auxílio de Matheus Yanko da Luz Aires, integrante do laboratório. Os materiais utilizados na produção foram: placa Arduino, acelerômetro e giroscópio, display oled, led e buzzer. Nas etapas da fabricação do produto teve-se a programação do acelerômetro, logo após encontrou-se a programação correta para o display oled, combinou-se as duas programações. E por fim, colocou-se um buzzer e um led como alertas visual e auditivo, e foi soldado tudo na placa de prototipagem.

\subsection{Descrição do processo}

Primeiramente, realizou-se uma pesquisa para compreender como se programava o acelerômetro MPU6050- figura 04 - visto que, o dispositivo ler três valores correspondentes aos eixos x,y e z, com isso realizou-se o cálculo para achar o vetor resultante dos eixos, após isto, no programa (software do arduino) foi aplicado a norma de vibração para o vetor resultante. Como o acelerômetro faz uma leitura contínua mesmo estando em uma posição parada- uma vez que está sujeito a aceleração da gravidade- converteu-se o valor do vetor resultante como equivalente a $g=9,81 \mathrm{~m} / \mathrm{s}^{2}$. Assim aplicou-se no programa mais uma variável, onde a aceleração sofrida pela atividade é o vetor resultante menos a aceleração da gravidade, desse modo o acelerômetro só indicará a aceleração quando colocado em movimento.

Na segunda etapa realizou-se a pesquisa para programação do display oled (figura 03) no Arduino. Reuniu-se a programação do acelerômetro com a do display, fazendo com que as informações do acelerômetro captadas pelo arduino (figura 02) possam ser enviadas para o display. Por conseguinte, incluiu-se um buzzer e um led (figura 06 e 05, respectivamente), para atuarem como alerta auditivo e visual, respectivamente.

A última etapa depois de toda a parte de programação finalizada, sucedeu-se a soldagem dos componentes eletrônicos na placa de prototipagem (figura 08), dado início ao circuito eletrônico de controle EPIN e assim, resultando no sensor visualizado na figura 07. 




Fonte: http://www.sermaker.com/arduini-nano-v3 (2019)

Figura 2 - Display Oled

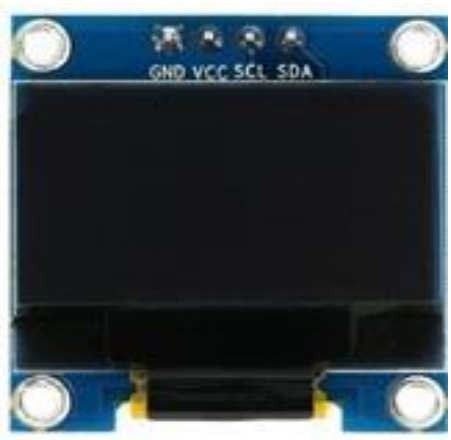

Fonte: https://produto.mercadolivre.com.br/MLB-708224500-display-oled-128x64-096-i2c-grafico-brancoarduino-_JM (2019)

Figura 3 - Acelerômetro e Giroscópio

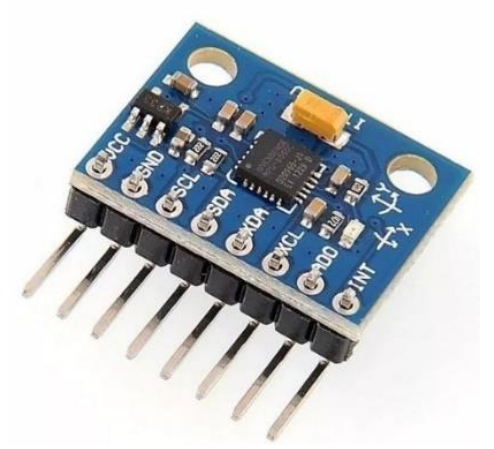

Fonte: <https://img.olx.com.br/images/36/365831099010352.jpg> (2019)

Figura 4 - Led 


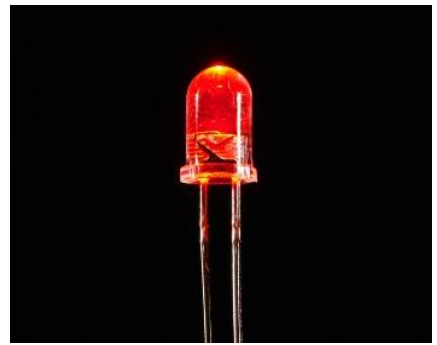

Fonte: <https://www.adafruit.com/product/297> (2019)

Figura 5 - Buzzer

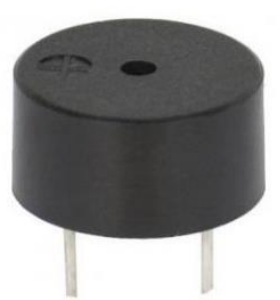

Fonte: http://www.roboliv.re/conteudo/buzzer/imagem (2019)

Figura 6 - Sensor Finalizado

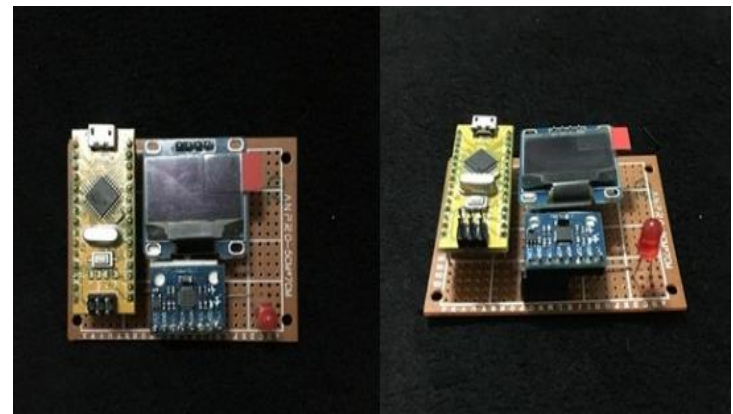

Fonte: Autoria própria (2018)

Figura 7 - Placa de prototipagem

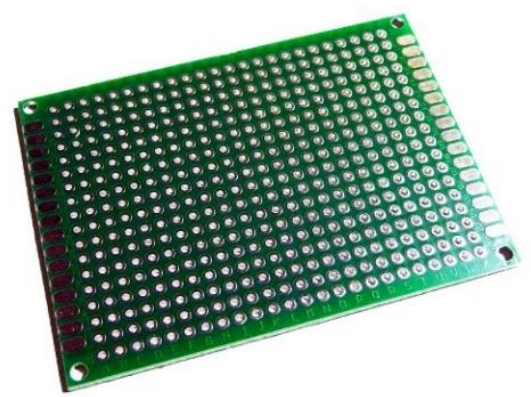

Fonte: < https://produto.mercadolivre.com.br/MLB-910024884-placa-5x7-cm-pre-furada-face-dupla-metalizdap-prototipagem-_JM?quantity=1> (2019)

\section{Resultados e discussão}


A proposta do EPIN de vibração inicialmente, vem da necessidade de informar e alertar o trabalhador a que nível de vibração localizada (mão e braço) ele está exposto, informação essa que pode ser usada pela gerência como ponto de partida para minimização da situação de risco quando se está acima do permitido pela NR. Seu princípio de funcionamento está associado a um circuito eletrônico que une um acelerômetro, display, buzzer e led a uma nano placa de arduino, que quando vinculado a luva antivibração permite a visualização da aceleração sofrida durante o trabalho, que também pode ser enviada para um supervisor quando emitido o sinal de alerta para o trabalhador.

\subsection{Descrição do produto}

O EPIN produzido foi visando a medição de vibração em diversas atividades que ofereçam este risco. Ele será combinado com a luva antivibração sendo acoplado na parte do dorso da mão, e medirá a aceleração em que o membro do operário estará submetido durante a realização de sua tarefa no trabalho e funciona à temperatura ambiente.

Quando se atinge uma vibração acima do indicado, como descrito na seção 2.4, é emitido um sinal sonoro e outro visual, assim ocorrendo é disparado um único bip e se a luz se acende, como sinal de alerta. Numa segunda ocorrência, é novamente disparado um bip juntamente com a luz. Mediante uma terceira ocorrência o bip passa a ser contínuo e a luz permanece acesa até ser dado uma pausa na atividade. Os dados de aceleração e temperatura do ambiente podem servir tanto como informações para o próprio trabalhador, como também podem ser transmitidas para a gerência, informando a situação em que o funcionário está exposto no seu ambiente de trabalho e assim, possa fazer uso de tais informações para desenvolver um plano de intervenção visando minimizar o risco à saúde durante a realização da tarefa.

O protótipo em questão foi colocado à prova com o objetivo de avaliar se de fato funcionava. Foram feitos vários testes e comprovado que o EPI opera de maneira esperada. Os testes foram realizados, em um primeiro momento, no laboratório de automação para constatar se havia falhas no seu funcionamento, e como não houve determinou-se que o protótipo atenderia as necessidades pensadas inicialmente, ou seja, serviria para alertar o trabalhador da velocidade a qual o mesmo está submetido. Em um segundo momento, após os testes iniciais, o modelo foi apresentado em sala de aula como um objeto de sucesso, fez-se demonstrações in loco comprovando a funcionalidade do EPI.

\section{Considerações finais}


Com a realização deste presente trabalho pudemos evidenciar a importância da relação Universidade/Empresa para o crescimento profissional e cidadão. Após o estudo sobre EPI, Normas Regulamentadoras, riscos ocupacionais- conceitos estes vistos no decorrer da disciplina Higiene e Segurança do Trabalho- foi possível entendermos as consequências causadas pela falta ou má utilização destes equipamentos, e assim pensarmos em algo que possa alertar o operário sobre o risco de ultrapassar o limite de tolerância permitido da máquina, no caso do artigo exposto máquina que apresente algum tipo de vibração.

As principais vantagens de se utilizar um EPIN estão relacionadas em prevenir acidentes ou possíveis danos à saúde do trabalhador, visto que, o EPIN servirá de alerta caso se ultrapasse o limite aceitável pela Norma, com isso o operário saberá o momento de parar a atividade ou diminuir o ritmo. Pode-se citar também que o funcionário se sentirá mais seguro e confiante em realizar o trabalho, em virtude de portar um dispositivo que lhe auxilie no ambiente de trabalho.

\section{REFERÊNCIAS}

ARDUINO. Disponível em: <https://www.arduino.cc/en/Guide/Introduction>. Acesso em: 06 Dez., 2018.

ATENUAÇÃO DE RUÍDO NA VENTILAÇÃO FORÇADA EM RESIDÊNCIAS: ANÁLISE EXPERIMENTAL DE UM PROTÓTIPO INOVADOR. Disponível em:

<https://www.researchgate.net/profile/Aloisio_Schmid/publication/283426714_Atenuacao_de_ruido_na_ventila cao_forcada_em_residencias_analise_experimental_de_um_prototipo_inovador/links/594847550f7e-9b1d 9 b2330 92/Atenuacao-de-ruido-na-ventilacao-forcada-em-residencias-analise-experimental-de-um-prototipoinovador.pdf\#page=53>. Acesso em: 03 Dez., 2018.

AZEVEDO, C. A. L.; SOBRAL, A. B. N.; AZEVEDO, E. G. F. D. EQUIPAMENTO ELETRÔNICO DE PROTEÇÃO INDIVIDUAL EPI INTELIGENTE - MÓDULO CAPACETE, Rio de janeiro. 1-9.

COMO FUNCIONAM ACELERÔMETROS E GIROSCÓPIOS. Disponível em:

<https://www.tecmundo.com.br/celular/4406-como-funcionam-acelerometros-e-giroscopios-.htm>. Acesso em: 06 Dez., 2018.

DA CUNHA, I.; GIAMPAOLI, E. Norma de higiene ocupacional- procedimento técnico: avaliação da exposição ocupacional a vibração em mãos e braços. São Paulo: Fundacentro, 2013.

ESTUDO DOS EFEITOS AUDITIVOS E EXTRA-AUDITIVOS DA EXPOSIÇÃO OCUPACIONAL A RUÍDO E VIBRAÇÃO. Disponível em: 〈http://www.scielo.br/pdf/\%0D/rboto/v68n5/a17v68n5.pdf>. Acesso em: 02 Dez., 2018.

FILHO, B.; NUNES, A. Segurança do trabalho \& gestão ambiental. 2011

MANUAL PRÁTICO DE AVALIAÇÃo E CONTROLE DE VIBRAÇÃo. Disponível em: <http://www.segurancanotrabalho.eng.br/sumario/indice_sumario_501584.pdf>. Acesso em: 02 Dez., 2018.

MATOS, U.; MÁSCULO, F. HIGIENE E SEGURANÇA DO TRABALHO. Recife: Ed. Escola Técnica Federal de Pernambuco, 1998.

Norma Regulamentadora 6. Disponível em: 〈http://www.guiatrabalhista.com.br/legislacao/nr/nr6.htm〉. Acesso em: 01 Dez., 2018.

PRINCÍPIOS DE HIGIENE E SEGURANÇA NO TRABALHO. Disponível em: <http://saber.unioeste.br/index.php/expectativa/article/viewFile/87/297>. Aceso em: 03 Dez., 2018.

ROZENFELD, H.; FORCELLINI, F.A.; AMARAL, D.C.; TOLEDO, J.C.; SILVA, S.L.; ALLIPRANDINI, D.H.; SCALICE, R.K. Gestão de Desenvolvimento de Produtos: uma referência para a melhoria do processo. São Paulo: Saraiva, 2006. 
SALIBA, T. Curso Básico de Segurança e Higiene Ocupacional. São Paulo: LTr, 2011.

SEGURANÇA, MEIO AMBIENTE E SÁUDE. Disponível em:

$<$ http://estudio01.proj.ufsm.br/cadernos/ifpe/tecnico_manutencao_automotiva/arte_seguranca.pdf >. Acesso em: 02 Dez., 2018. 